ISSN 0001-6002/2002/44/2/74-78

A cta M édica Costarricense, $\bigcirc 2002$

Colegio de Médicos y Cirujanos

\title{
Resultados de la categorización de una muestra de pacientes con diagnóstico de egreso de esquizofrenia
}

Ana Patricia Montero',2,3, Teresa Balderas ${ }^{4}$, Mariana Pereira', Arturo Lizano', ${ }^{1,5}$ Regina Armas $^{6}$, Rolando Medina ${ }^{4}$, Salvador Contreras ${ }^{4}$, Albana Dassari ${ }^{4}$, Israel Katz ${ }^{7}$, Doug Levinson ${ }^{8}$, Jean Jacques Garbarz ${ }^{9}$, Michael Escamilla ${ }^{4}$, Henriette Raventós ${ }^{1,10}$,

R esumen:La esquizofrenia es una enfermedad que afecta aproximadamente al $1 \%$ de la población mundial. Es una de las enfermedades psiquiátricas más debilitantes con un alto costo económico, social, familiar y personal. En Costa Rica, la esquizofrenia constituye la primera causa de internamiento entre los desórdenes psiquiátricos en el Hospital Nacional Psiquiátrico. El presente estudio analiza el diagnóstico preliminar de los primeros 120 pacientes incluidos en el proyecto sobre la Genética de la Esquizofrenia. Al comparar el diagnóstico del entrevistador, el diagnóstico final por consenso y el diagnóstico del último egreso hospitalario se encontraron diferencias en alrededor del $40 \%$ de los sujetos en el estudio. Se encontró un relativo sub-registro de la sintomatología afectiva en los diagnósticos de egreso con el consecuente sobre-diagnostico de los trastornos esquizofrénicos en los hospitales nacionales. El trastorno esquizoafectivo fue uno de los diagnósticos con menor concordancia. Las diferencias diagnósticas encontradas pueden ser parcial mente explicadas por el uso de pautas diagnósticas diferentes y/o una mayor rigurosidad diagnóstica utilizada en investigación que no es necesaria en la clínica. Sin embargo, la concurrencia de diferentes síntomas en las enfermedades psiquiátricas severas y las limitaciones impuestas por la categorización diagnóstica, obliga al psiquiatra a encasillar al paciente en una categoría que puede no representar completamente la enfermedad de ese paciente. El contar con marcadores biológicos en un futuro podría servir para clasificar estos síndromes en entidades diagnósticas más precisas, a explicar la fisiopatología de estas enfermedades y mejorar el tratamiento.

Pal abras clave: Esquizofrenia, pautas diagnósticas.

Recibido: 05 de marzo del 2002.

Aceptado: 23 de abril del 2002

\begin{abstract}
A breviaturas: HNP: Hospital Nacional Psiquiátrico, UCR: Universidad de Costa Rica; SC: esquizofrenia; EP: esquizofrenia paranoide; $\mathrm{ECl}$ : esquizofrenia crónica indiferenciada; ER: esquizofrenia residual; $B P$ Desorden Bipolar; TEA: trastorno esquizoafectivo; DM : depresión mayor; DIGS: entrevista diagnóstica para estudios genéticos; FIGS: entrevista familiar para estudios genéticos; MED: mejor estimado diagnóstico; DUE: diagnóstico del último egreso; DSM: manual de diagnóstico para enfermedades mentales; CIE: clasificación internacional de enfermedades.

Centro de Investigación en Biología Celular y M olecular, U niversidad de Costa Rica.

Escuela de M edicina, Universidad de Costa Rica.

Hospital Nacional Psiquiátrico, Caja Costarricense del Seguro Social.

Centro de Ciencias de la Salud de la Universidad de Texas en San Antonio.

Hospital Cima San José.

Departamento de Psiquiatría, Universidad de California, San Francisco Hospital General de San Francisco, San Francisco, California.

Universidad de Pennsylvania, Philadelphia, Pennsylvania.

Departmento de Salud M ental de San Francisco, California.

Escuela de Biología, Universidad de Costa Rica.
\end{abstract}

Correspondencia: Henriette Raventós, CIBCM , Ciudad de la Investigación, Universidad de Costa Rica, San Pedro de M ontes de Oca, Fax: 207-3190, hravento@ racsa.co.cr
La esquizofrenia (SC) es una enfermedad que afecta aproximadamente al $1 \%$ de la población mundial. Es una de las enfermedades psiquiátricas más debilitantes con un alto costo económico, social, familiar y personal ${ }^{1,2}$. En Costa Rica, aunque no se cuentan con datos epidemiológicos, un 20\% de los internamientos del Hospital $\mathrm{N}$ acional Psiquiátrico son por esquizofrenia, lo que constituye la primera causa de internamiento entre los desórdenes psiquiátric $\cos ^{3-6}$. En el año 2000, estos internamientos consumieron el 35\% de los días estancia del Hospital Nacional Psiquiátrico lo que representa un costo aproximado a los 1,000,000,000 (mil millones de colones) solo en el HNP ${ }^{6}$. Este monto no incluye el costo por consulta externa, medicamentos, pago de incapacidades temporales o permanentes, ingresos no percibidos, ni los costos emocionales no cuantificables para la familia y el paciente.

EI diagnóstico de esquizofrenia es uno de los grandes retos de la psiquiatría moderna. Los avances en el conocimiento biológico y el desarrollo de nuevos sistemas de clasificación en psiquiatría, han modificado poco la dicotomía definida por 
K raepelin a principios del siglo pasado entre la "dementia precox" y la "locura maniaco depresiva ${ }^{7-9}$.

El complejo síndrome de la esquizofrenia puede incluir alteraciones clásicas como delirios, alucinaciones y pensamiento, lenguaje y comportamiento desorganizado ${ }^{8}$. Las manifestaciones clínicas pueden además variar en el tiempo y combinarse en ocasiones con síntomas afectivos (maniacos o depresivos), trastornos de ansiedad, problemas legales y adicción a alcohol y/o drogas ilícitas. La dificultad para clasificar a los pacientes con psicosis en entidades clínicas precisas solo ha sido parcialmente resuelta con la evolución de los manuales diagnósticos ${ }^{7-9}$.

El presente estudio presenta un análisis diagnóstico preliminar de los primeros 120 pacientes incluidos en el proyecto "Localización de genes de susceptibilidad a la esquizofrenia en la población costarricense". Se compara el diagnóstico del entrevistador (DIGS), el diagnóstico por consenso (MED) y el diagnóstico del último egreso (DUE) y se discuten algunas posibles causas para las diferencias encontradas.

\section{Materiales y Métodos}

Este estudio fue aprobado por los comités de investigación (éticos 0 éticos-científicos) del Ministerio de Salud, Universidad de Costa Rica, Hospital Nacional Psiquiátrico, Universidad de California en San Francisco y Centro M édico de Ciencias de la Salud de la U niversidad de Texas en San A ntonio. Los criterios de inclusión fueron: (1) al menos un internamiento en psiquiatría, (2) al menos un diagnóstico de egreso por esquizofrenia, (3) edad de inicio antes de los 40 años y (4) al menos 3 de 4 apellidos de origen en el Valle Central de Costa Rica. Todos los pacientes fueron entrevistados entre abril de 1997 y octubre de 2000. LoS sujetos fueron referidos por especialistas que laboran en diferentes hospitales nacionales.

\begin{tabular}{|lrrrrrrr|}
\hline \multicolumn{7}{|c|}{$\begin{array}{c}\text { Cuadro 1. } \\
\text { Diagnóstico del último egreso (DUE) de los } \\
\text { pacientes incluidos en el estudio }\end{array}$} \\
\hline Diagnóstico & HOMBRES & MUJERES & TOTAL \\
\hline & $\mathrm{N}$ & $(\%)$ & $\mathrm{N}$ & $(\%)$ & $\mathrm{N}$ & $(\%)$ \\
Esquizofrenia & 55 & $(78.6)$ & 34 & $(68.0)$ & 89 & $(74.2)$ \\
Trastorno esquizoafectivo & 8 & $(11.4)$ & 11 & $(22.0)$ & 19 & $(15.8)$ \\
Desorden Bipolar & 0 & $(0.0)$ & 1 & $(2.0)$ & 1 & $(0.8)$ \\
Depresión Mayor con & & & & & & \\
psicosis & 0 & $(0.0)$ & 1 & $(2.0)$ & 1 & $(0.8)$ \\
Psicosis no especificada & 3 & $(4.3)$ & 1 & $(2.0)$ & 4 & $(3.3)$ \\
Otros diagnósticos & 4 & $(5.7)$ & 2 & $(4.0)$ & 6 & $(5.0)$ \\
\hline TOTAL & 70 & $(100)$ & 50 & $(100)$ & 120 & $(100)$ \\
& & & & & & \\
\hline
\end{tabular}

Previo consentimiento informado del paciente y un familiar cercano o testigo, el paciente fue entrevistado por un psiquiatra bilingüe y ciego al diagnóstico, utilizando la Entrevista Diagnóstica para Estudios Genéticos (DIGS) ${ }^{10}$. Todos los entrevistadores fueron entrenados en el uso de este instrumento por personal calificado. La conducción de esta entrevista duró entre 2 y 3 horas en promedio. Una vez finalizada, el entrevistador resumió la información en una historia clínica o narrativa y llegó a un diagnóstico presuntivo. A demás, se entrevistó a un familiar cercano con la Entrevista Familiar para Estudios Genéticos (FIGS). La información del expediente clínico del paciente fue resumida por personal médico utilizando otro instrumento semiestructurado previamente diseñado. La información completa (DIGS, narrativa, FIGS y expediente clínico) fue analizada por dos especialistas en esquizofrenia en forma independiente y luego en forma conjunta para alcanzar un diagnóstico único de consenso o Mejor Estimado Diagnóstico (MED), utilizando las pautas del DSM IV. La confiabilidad entre entrevistadores y estimadores ha sido analizada $^{11}$. Ocasionalmente fue necesario realizar nuevas visitas a los pacientes y familiares para completar información adicional solicitada por los estimadores a cargo del MED. Los casos en que no fue posible alcanzar un consenso 0 un diagnóstico presuntivo, fueron clasificados como psicosis no especificada. El trastorno esquizoafectivo (TEA) no fue subdividido en subtipos porque es poco frecuente encontrar esta clasificación en los diagnósticos de egreso (solo 3 de 23 fueron clasificados).

\section{Resultados}

En el presente estudio, se analiza la información clínica de los primeros 120 sujetos (50 mujeres y 70 hombres) con Mejor Estimado Diagnóstico (MED) incluidos en el estudio de la genética de la esquizofrenia que se realiza en la U niversidad de Costa Rica. Todos ellos tuvieron al menos un diagnostico de egreso de esquizofrenia (cualquier tipo) en un servicio hospitalario de psiquiatría durante la evolución de su enfermedad. El diagnóstico del último egreso (DUE) fue de esquizofrenia en $71 \%$ y trastorno esquizoafectivo en $19 \%$; el $10 \%$ restante tuvo otros diagnósticos en su último egreso.

Los tipos de esquizofrenia más frecuentes por DUE de la muestra en estudio fueron esquizofrenia paranoide (EP) esquizofrenia crónica indiferenciada $(E C I)$ y esquizofrenia residual (ER). En el Cuadro 2, se compara la relación porcentual de cada subtipo entre la muestra en estudio en relación a los egresos del Hospital Nacional Psiquiátrico (HNP) para el año 2000. Los egresos por esquizofrenia del HNP se han mantenido entre 700 y 800 por año desde 1997 siendo los tipos más frecuentes la ER (48-57\%) seguida de la EP $(40-45 \%)^{3-6}$.

La Figura 1 compara la distribución de los sujetos por el diagnóstico por último egreso (DUE), el diagnóstico del entrevistador (DIGS) y el MED. Por MED, el 62\% (75/120) de los sujetos fueron diagnosticados con alguno de los tipos de esquizofrenia en comparación a 44\% (53/120) por DIGS y 


\begin{tabular}{|lcc|}
\hline \multicolumn{3}{|c|}{ Cuadro 2} \\
\multicolumn{3}{|c|}{ Distribución porcentual por diagnóstico de } \\
egreso entre (a) los sujetos en estudio con DUE \\
por esquizofrenia y (b) los egresos totales del \\
HNP por esquizofrenia para el año & 2000 (3) \\
\hline \multicolumn{4}{|c}{} & Sujetos & Egresos \\
\hline Esquizofrenia Paranoide & 48.2 & 42.8 \\
Esquizofrenia Indiferenciada & 24.7 & 2.9 \\
Esquizofrenia Residual & 16.5 & 48.6 \\
Esquizofrenia Hebefrénica & 5.9 & 3.2 \\
Esquizofrenia Catatonica & 2.4 & 0.0 \\
Otros, no esp. & 2.4 & 2.5 \\
\hline
\end{tabular}

$71 \%(85 / 120)$ por DUE. Para los trastornos afectivos, solo 1 caso fue diagnosticado por último egreso como bipolar (BP) y 1 como depresión mayor (DM ) en comparación a 12 y 6 por MED. Por el contrario, el entrevistador tiende a diagnosticar menos casos de esquizofrenia y más casos de trastornos esquizoafectivos (TEA) o afectivos en comparación al MED: 32 TEA en comparación a 23, 14 BP en comparación a 12 y 8 DM en comparación a 6. En un 11\% (13/120) de los casos, el entrevistador no pudo llegar a un diagnóstico con solo la información de la entrevista al paciente por lo que lo clasificó como psicosis no especificada.

L a concordancia diagnóstica para cada sujeto en estudio entre el DIGS y MED es del $68 \%$ y entre el DUE y el MED de un $57 \%$. Sin embargo, es necesario enfatizar que el MED y el DIGS se basan en las pautas diagnósticas del DSMIV mientras que el DUE en el CIE 9 o 10 . La mayor concordancia entre el MED y el DIGS se presenta para los diagnósticos de esquizofrenia paranoide, desorden bipolar y depresión mayor. El diagnóstico con menor concordancia es el de trastorno esquizoafectivo. La concordancia entre el diagnóstico del entrevistador y el MED es mayor para hombres $(71.2 \%)$ que para mujeres $(64.0 \%)$.

En el cuadro 3 se muestra el diagnóstico del entrevistador en los casos con MED de esquizofrenia, trastorno esquizoafectivo 0 trastorno afectivo (que incluye desorden bipolar y depresión mayor). No se comparó el diagnóstico del hospital por dos razones: el hospital utiliza otras pautas diagnósticas y es de esperar que exista menor concordancia debido a que la selección de la muestra no fue aleatoria.

La mayor concordancia se observa en el grupo de pacientes $(n=18)$ que fueron diagnosticados con un trastorno afectivo (bipolar o depresión mayor) por el MED (77\%). Este porcentaje se eleva a un $94 \%$ si se suman los casos que el entrevistador consideró como trastorno esquizoafectivo. Solo en uno de los sujetos de este grupo, el entrevistador no llegó a un diagnostico con la entrevista. En ninguno de los

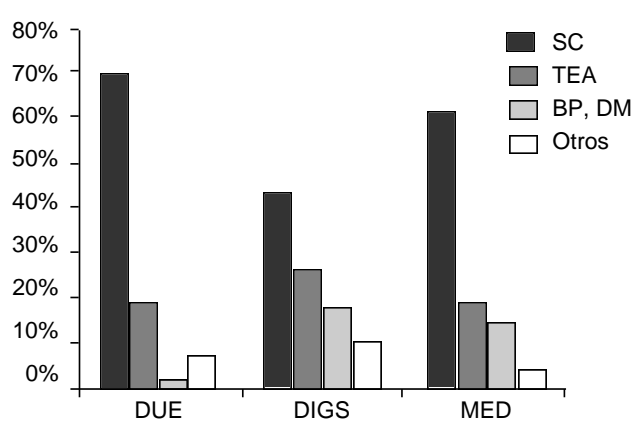

Figura 1: Comparación entre el diagnóstico del último egreso (DUE), el diagnóstico del entrevistador (DIGS) y el diagnóstico por consenso (MED).

casos afectivos por MED, el entrevistador diagnosticó esquizofrenia 0 un trastorno psiquiátrico sin componente afectivo.

La concordancia entre el MED y el DIGS en los sujetos con un diagnóstico de esquizofrenia $(n=75)$ o de trastorno esquizoafectivo $(n=23)$ por MED fue similar $(68 \%$ y $65 \%$ respectivamente). Sin embargo, en los casos con diagnóstico de trastorno esquizoafectivo por MED, el entrevistador no logró llegar a un diagnóstico con la entrevista en 5 casos (22\%) y en 3 casos (13\%) dio un diagnostico afectivo. En ninguno de los casos esquizoafectivos, el entrevistador diagnosticó esquizofrenia 0 un trastorno psiquiátrico sin componente afectivo.

Solo en 4 sujetos (5\%) con un diagnóstico de esquizofrenia por MED, el entrevistador diagnosticó trastorno bipolar ${ }^{3} 0$ depresión mayor con psicosis ${ }^{1}$. En 6 sujetos (8\%) no logró al canzar un diagnóstico con la entrevista. En el restante $87 \%$, el entrevistador diagnosticó esquizofrenia o trastorno esquizoafectivo. Sin embargo, si se separa este grupo con MED de esquizofrenia por sexo, se observa una mayor tendencia a dar un diagnóstico afectivo (trastorno esquizoafectivo, desorden bipolar o depresión mayor) a mujeres (11/25, 2 B P, 9 TEA) que a hombres (7/50, 1 BP, 1 DM , 5 TEA) por parte del entrevistador.

\section{Cuadro 3}

Diagnóstico del entrevistador en los sujetos con mejor estimado diagnóstico de esquizofrenia, trastornos afectivos (bipolar/depresión mayor) y trastorno esquizoafectivo.

\begin{tabular}{lrrrrrr} 
Diagnóstico & \multicolumn{2}{c}{$\begin{array}{c}\text { Esquizofrenia } \\
\text { por }\end{array}$} & \multicolumn{2}{c}{ T. Afectivos por } & \multicolumn{2}{c|}{ T. Esquizoafectivo } \\
& $\mathrm{N}$ & $(\%)$ & $\mathrm{N}$ & $(\%)$ & $\mathrm{N}$ & $(\%)$ \\
\hline Esquizofrenia & 51 & $(68.0)$ & 0 & $(0.0)$ & 0 & $(0.0)$ \\
Trastornos Afectivos (BP/DM) & 4 & $(5.3)$ & 14 & $(77.8)$ & 3 & $(13.0)$ \\
Trastorno Esquizoafectivo & 14 & $(18.7)$ & 3 & $(16.7)$ & 15 & $(65.2)$ \\
Otros, no esp. & 6 & $(8.0)$ & 1 & $(5.6)$ & 5 & $(21.7)$ \\
\hline Total & 75 & $(100.0)$ & 18 & $(100.0)$ & 23 & $(100.0)$ \\
\hline
\end{tabular}




\section{Discusión}

El presente estudio muestra semejanzas y diferencias entre los diagnósticos hospitalarios, del entrevistador y del equipo de expertos, interesantes de analizar. Sin embargo, primero hay que señalar las limitaciones del presente análisis. La selección de esta muestra no fue al eatoria. La distribución por tipo de esquizofrenia entre la muestra en estudio versus los egresos anuales en el Hospital Nacional Psiquiátrico evidencia que la presente muestra no es representativa de los egresos por esquizofrenia del HNP. Además, hay que considerar que el diagnóstico hospitalario se basa en pautas diagnósticas diferentes a las utilizadas por el MED y el entrevistador. Finalmente, el equipo de entrevistadores está compuesto por psiquiatras de diferentes formaciones que aunque hayan sido entrenados en el uso del instrumento diagnóstico, su formación puede influir en su interpretación personal de las manifestaciones clínicas y pautas diagnósticas. Por no ser una muestra aleatoria de todos los diagnósticos psiquiátricos (ni siquiera de los egresos por esquizofrenia) y utilizar pautas diagnósticas diferentes, no permite hacer un análisis del diagnóstico hospitalario en el país. Sin embargo, si permite llegar a algunas conclusiones y posibles explicaciones e ilustra al gunas de las dificultades del diagnóstico psiquiátrico.

En primer lugar, alrededor del $40 \%$ de los sujetos en el estudio fueron diagnosticados en categorías diferentes por el mejor estimado diagnóstico y por el diagnóstico de su último egreso. Esta diferencia puede ser parcialmente explicada por el uso de pautas diagnósticas diferentes y/o una mayor rigurosidad diagnóstica utilizada en investigación que no es necesaria en la clínica. Por ejemplo, los casos que el MED diagnosticó como una psicosis no especificada y que contaban con un diagnóstico específico de egreso, se pueden explicar a que los estimadores son más rigurosos con las pautas diagnósticas para no incluir en el análisis genético individuos con otros diagnósticos. Esta rigurosidad no se justifica en los servicios hospitalarios en los que es importante llegar a un diagnóstico, aunque sea presuntivo, para iniciar el tratamiento correspondiente.

A demás, el diagnóstico diferencial entre depresión, síntomas negativos y extrapiramidales (en especial, akinesia) en un paciente esquizofrénico hospitalizado puede ser difícil ${ }^{12}$. En este estudio, existe un grupo de pacientes que el MED diagnosticó con desorden bipolar $(n=12)$ o depresión mayor $(n=6)$ cuyo diagnóstico de último egreso fue de esquizofrenia $(n=9)$ o trastorno esquizoafectivo $(n=9)$. Por lo anterior, los síntomas afectivos no se reflejaron en el diagnóstico de egreso en la mitad de estos casos (en los 9 con diagnóstico de esquizofrenia). Sin embargo, es importante mencionar que en algunos de estos casos fue posible documentar el uso de medicamentos para sus síntomas afectivos, aunque el diagnóstico no reflejara la presencia de dichos síntomas.

La presencia de síntomas afectivos es a menudo percibida como una manifestación de la esquizofrenia, más que de un trastorno afectivo particular. Esta noción probablemente se origina de la dicotomía propuesta por K raepelin, entre las psicosis afectivas y las psicosis esquizofrénicas. B orrows ${ }^{13}$ estudió la base de datos del National Institute of Mental Health Epidemiological Catchment Area, en la cual se entrevistaron a 20.000 personas de 18 a 65 años con el Diagnostic Interview Schedule. En esta base, se encontró una prevalencia de vida de $1.5 \%$ de esquizofrenia y una co-morbilidad con trastornos del afecto con una razón de probabilidad de 14 (el gold standard es de 10). Por tanto, existe la posibilidad de que un esquizofrénico presente un episodio depresivo mayor en algún momento de su vida. También se encontró una alta comorbilidad con el trastorno bipolar. EI NCS ${ }^{14}$, otra base de datos que estudió a 8.000 individuos con esquizofrenia, encontró una prevalencia de trastornos depresivos unipolares en un 59\% de estas personas, depresión mayor y distimia en un $27 \%$ y trastorno depresivo menor en un $10 \%$. A proximadamente el $22 \%$ de los pacientes esquizofrénicos pueden tener un diagnóstico de trastorno bipolar en al gún momento de su vida.

En contraposición al relativo sub-registro de sintomatología afectiva en los diagnósticos de egreso en esta muestra, los entrevistadores tienden a sobre-diagnosticar los trastornos afectivos. Esto puede reflejar el estilo individual de los entrevistadores y/o una tendencia a sobre-valorar los posibles diagnósticos de exclusión de la esquizofrenia para no incluir pacientes con otros diagnósticos en el análisis genético. También puede reflejar la percepción del paciente sobre su enfermedad (con un mayor énfasis a los síntomas afectivos), mientras que el hospital tiende a enfatizar los síntomas sicóticos. Llama la atención la mayor concordancia entre el MED y el diagnóstico del entrevistador en pacientes masculinos y la tendencia a sobre-diagnosticar trastornos afectivos en mujeres. Una posible explicación sería que las mujeres expresan más síntomas afectivos que los hombres con esquizofrenia durante la entrevista, lo cual podría ser el resultado de diferencias de género aprendidas, 0 a que los psiquiatras tienden a sobre-val orar los síntomas afectivos en mujeres pero no en hombres. Este hallazgo, aunque requiere confirmación y estudios posteriores, no ha sido reportado por otros investigadores.

Finalmente, el trastorno esquizoafectivo es uno de los diagnósticos con menor concordancia entre el MED, el DUE y el entrevistador. Esto no es sorprendente ya que es una categoría diagnóstica relativamente nueva cuyos pautas e interpretación varía entre sistemas diagnósticos. En esta categoría concurren síntomas de esquizofrenia y de enfermedad bipolar y es posible que los pacientes sean catalogados como bipolares, esquizoafectivos 0 esquizofrénicos dependiendo de las pautas diagnósticas utilizadas y su interpretación. En el DSM IV por ejemplo, no se especifica que tan importantes deben ser los síntomas afectivos en la historia longitudinal de un paciente con cuadros sicóticos no afectivos para que sea catal ogado como esquizoafectivo versus esquizofrénico. Para los fines de este estudio, el trastorno esquizoafectivo se diagnostica si los síntomas afectivos están presentes en al menos un 30\% del total de la duración de la enfermedad, incluyendo todas las 
crisis. Sin embargo, aunque esta recomendación parte del grupo que desarrolló las pautas del DSMIV, no está especificada en ellas (comunicación personal).

El trastorno esquizoafectivo, además de ser difícil de categorizar, es una entidad interesante ya que cuestiona la validez de la dicotomía propuesta por K raepelin entre la esquizofrenia y la enfermedad bipolar. Si estas categorías diagnósticas fueran totalmente independientes, se esperaría una menor prevalencia del trastorno esquizoafectivo. Otros resultados han contribuido a cuestionar esta dicotomía. Estudios familiares muestran un mayor riesgo de trastornos afectivos en familiares de un probando esquizofrénico y un mayor riesgo de esquizofrenia en familiares de un probando con un trastorno bipolar. Finalmente, es interesante indicar que los estudios genéticos han encontrado algunos loci de susceptibilidad genética comunes entre ambas enfermedades ${ }^{15}$.

EI presente estudio evidencia algunas de las dificultades para llegar a un diagnóstico en las enfermedades psiquiátricas severas. La comparación entre diagnósticos muestra que el obtener información adicional del resumen completo de los registros médicos y de la familia, mejora la posibilidad de al canzar un diagnóstico. El conducir una entrevista semiestructurada cuando el paciente no está en un servicio de emergencias también puede contribuir a alcanzar un diagnóstico más preciso. Sin embargo, la concurrencia de diferentes síntomas en las enfermedades psiquiátricas severas y las limitaciones impuestas por la categorización diagnóstica, obliga al psiquiatra a encasillar al paciente en una categoría que puede no representar completamente la enfermedad de ese paciente. El contar con marcadores biológicos en un futuro podría servir para clasificar estos síndromes en entidades diagnósticas más precisas, a explicar la fisiopatología de estas enfermedades y a orientar el mejor esquema terapéutico

\section{Abstract}

A pproximately $1 \%$ of the world population is affected with schizophrenia. It is one of the most debilitating psychiatric disorders with a high economic, social, familial and personal cost. In Costa Rica, it is the first cause of hospitalization among psychiatric disorders at the National Psychiatric Hospital. This study analyses the diagnosis of the first 120 subjects included in the Genetic Study of Schizophrenia. A pproximately $40 \%$ of the cases were discordant for the diagnosis when comparing the diagnosis of the interviewer, the final consensus diagnosis and the last discharge diagnosis. The last hospital discharge diagnosis showed a relative sub-registry of affective symptoms and an overdiagnosis of schizophrenic disorders. The diagnosis of schizoaffective disorders was the least concordant. The differences found could be partly explained by the use of different diagnostic criteria and/or by a more rigorous application of these criteria in research. Nonetheless, the concurrence of different symptoms in the severe mental disorders and the limitations imposed by strict diagnostic criteria, might give a diagnosis to a patient which doesn't completely represent his/her illness. The future availability of biological markers might help to classify these disorders in more precise clinical entities, to better understand the physiopathology and improve treatment.

\section{Referencias}

1. Wyatt RJ, Henter I, Leary M C, Taylor E. An economic evaluation of schizophrenia. Soc-Psychiatry-Psychiatr-E pidemiol 1995; 30(5):196205.

2. "Schizophrenia", in Comprehensive Textbook of Psychiatry, 7th Edition, B enjamín I. Sadock, Virginia A. Sadock, Lippincott Williams and Williams, (version CDRoom), 1999.

3. Departamento de Registros Médicos del Hospital Nacional Psiquiátrico. Anuario Estadístico Año 1997. Caja Costarricense del Seguro Social.

4. Departamento de Registros Médicos del Hospital Nacional Psiquiátrico. A nuario Estadístico A ño 1998. Caja Costarricense del Seguro Social.

5. Departamento de Registros Médicos del Hospital Nacional Psiquiátrico. Anuario Estadístico Año 1999. Caja Costarricense del Seguro Social.

6. Departamento de Registros Médicos del Hospital Nacional Psiquiátrico. A nuario Estadístico A ño 2000. Caja Costarricense del Seguro Social.

7. DSM III R, Diagnostic and Statistical Manual of Mental Disorders, Published by the A merican Psychiatric A ssociation, 1987

8. DSM IV , M anual diagnóstico y Estadístico de los Trastornos M entales, A merican Psychiatric A ssociation, Publicado por M asson SA, 1995

9. CIE-10, Clasificación Internacional de Enfermedades, (versión en Español), publicado por la OM S, 1998.

10. N urnberger II $\mathrm{r}$, B lehar M C, K aufmann CA, Y ork-Cooler C, Simpson SG, Harkavy-Friedman J et al. Diagnostic Interview for Genetic Studies. Rationale, unique features, and training. NIMH Genetics Initiative. A rch Gen Psychiatry 1994; 51: 849-59.

11. M edina, RA, Escamilla, MA, Armas, R, Levinson, D, Dassori A, Contreras $\mathrm{S}$, Raventos $\mathrm{H}$. Diagnostic Reliability in a Study of Schizophrenic (SC) Subjects from the Costa Rican Population. Am J M ed Genet 2001;105(7): 599

12. Fleicshhacker W. Differential diagnosis in Schizophrenia. J Clin Psych 1998; 16: 5-7.

13. Burrows G. Post Psychotic Depression. J Clin Psych 1998; 16: 4-5.

14. de A ndreasen N. M ood disorders and schizophrenia. J Clin Psych 1988; 16: $7-8$

15. Bramon E y Sham PC. The common genetic liability between schizophrenia and bipolar disorder: a review. Curr Psychiatry Rep 2001; 4:332-7.

\section{Agradecimientos}

Al personal del Departamento de Registros del Hospital Nacional Psiquiátrico por su colaboración. Este estudio es financiado por (1) la ICGEB (Contracto No. 98/030) (2) Instituto Nacional de Salud M ental de los Estados Unidos (CONTRATO \# K-647-1-17 con UTHSCSA) y (3) la Vicerrectoría de Investigación de la Universidad de Costa Rica (801-98-500). 